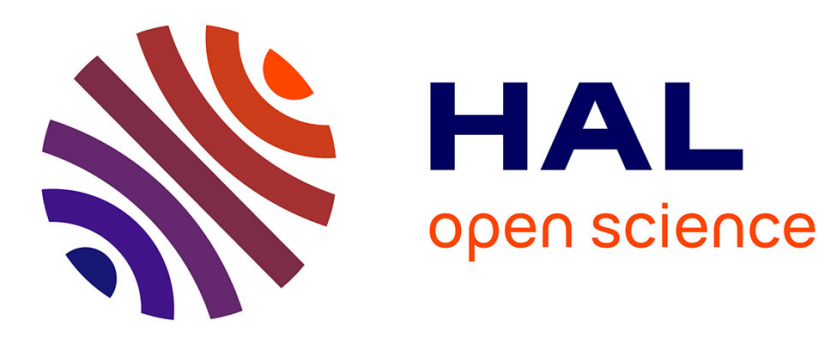

\title{
Une nouvelle structure pour un laser N2
}

\author{
G. Delouya, P. Leprince, H. Milleon
}

\section{To cite this version:}

G. Delouya, P. Leprince, H. Milleon. Une nouvelle structure pour un laser N2. Revue de Physique Appliquée, 1977, 12 (6), pp.969-973. 10.1051/rphysap:01977001206096900 . jpa-00244268

\section{HAL Id: jpa-00244268 https://hal.science/jpa-00244268}

Submitted on 1 Jan 1977

HAL is a multi-disciplinary open access archive for the deposit and dissemination of scientific research documents, whether they are published or not. The documents may come from teaching and research institutions in France or abroad, or from public or private research centers.
L'archive ouverte pluridisciplinaire HAL, est destinée au dépôt et à la diffusion de documents scientifiques de niveau recherche, publiés ou non, émanant des établissements d'enseignement et de recherche français ou étrangers, des laboratoires publics ou privés. 


\title{
UNE NOUVELLE STRUCTURE POUR UN LASER $\mathbf{N}_{2}(*)(* *)$
}

\author{
G. DELOUYA, P. LEPRINCE et H. MILLEON \\ Laboratoire de Physique des Plasmas (***), Bâtiment 212 \\ Université Paris XI, Centre d'Orsay 91405 Orsay, France
}

(Reçu le 22 décembre 1976, accepté le 17 mars 1977)

\begin{abstract}
Résumé. - Compte tenu d'une étude du circuit électrique de la décharge d'un laser $\mathrm{N}_{2}$ (circuit Blumlein) une nouvelle structure a été mise au point ; quelques résultats expérimentaux sont présentés. Une explication des courbes donnant l'énergie laser en fonction de la pression est donnée : le maximum d'énergie est obtenu lorsque $\frac{\mathrm{d} V_{\mathrm{k}}}{\mathrm{d} t}\left(V_{\mathrm{k}}=V_{\mathrm{c}}\right)$ est maximum ( $V_{\mathrm{k}}$ tension aux bornes de la décharge, $V_{\mathrm{c}}$ tension de claquage).

Abstract. - Based on the results obtained with the equivalent circuit of a transversely excited $\mathrm{N}_{2}$ laser (Blumlein circuit), a new simple structure has been constructed. Preliminary experimental results are presented. An explanation of the curves providing variation of laser energy output with pressure is given. The maximum power is obtained when the voltage slope $\frac{\mathrm{d} V_{\mathrm{k}}}{\mathrm{d} t}\left(V_{\mathrm{k}}=V_{\mathrm{c}}\right)$ is maximum ( $V_{\mathrm{k}}$, voltage drop across the gap; $V_{\mathrm{c}}$, breakdown voltage).
\end{abstract}

1. Introduction. - Le laser $\mathrm{N}_{2}$ ne peut fonctionner qu'en impulsion [1] en raison de la durée de vie du niveau supérieur $C^{3} \Pi_{u}$ (quelques dizaines de ns pour des pressions de 20 à 100 torr). Pour cette raison, on a réalisé des décharges transverses fonctionnant en impulsion permettant d'obtenir un milieu amplificateur de plusieurs dizaines de centimètres de long tout en ayant des temps de formation de la décharge très courts. En raison de l'intérêt que présente ce type de décharge non seulement pour les lasers $\mathrm{N}_{2}$ mais aussi pour d'autres types de lasers : $\mathrm{KrF}, \mathrm{XeF}$ [3], une étude électrique de la structure semble nécessaire pour comprendre le fonctionnement de la décharge. Dans cet article, après une étude de la structure type Blumlein, une nouvelle structure est proposée ; tout en gardant des performances tout à fait correctes (rendement de 0,1 à $0,3 \%$ ), sa fiabilité est meilleure, le laminé n'étant chargé que pendant le fonctionnement du laser. Dans une dernière partie, une explication des courbes de l'énergie laser en fonction de la pression est donnée en considérant l'étude des transferts d'énergie dans la structure.

(*) Communication présentée au Congrès National de Physique des Plasmas, Paris, 6-10 décembre 1976.

(**) Recherches partiellement financées par la D. R. M. E. (***) Laboratoire associé au C. N. R. S.
2. Etude électrique des structures. - La structure la plus simple employée actuellement est la suivante : la cavité laser comprenant les deux électrodes est insérée dans un circuit du type Blumlein (Fig. 1), constitué par une ligne plate (laminé ou microstrip). Les deux parties du circuit étant chargées, on courtcircuite l'une d'elles (point C) à l'aide d'un éclateur ; une onde de tension se propage le long de la première ligne (de $\mathrm{C}$ vers $\mathrm{A}, \mathrm{B}$ ) initie la décharge et par suite génère une autre onde dans l'autre ligne. La tension aux bornes de la décharge est donnée par la superposition de ces deux ondes. On peut choisir la position du court-circuit de manière à avoir la décharge d'abord en $A$ et qu'ensuite le front d'ionisation se propage vers $B$ avec une vitesse voisine de celle de la lumière. Une solution pour obtenir exactement la vitesse de la lumière est d'utiliser une ligne en forme de parabole avec le court-circuit en son foyer [2].

Tout ceci n'est évidemment valable que si le temps de montée $\tau_{\mathrm{m}}$ de l'onde de tension est petit devant le temps de propagation $\tau_{\mathbf{p}}$ :

- $\tau_{\mathrm{p}}$ est donné par $l / v_{\mathrm{p}}$ où $v_{\mathrm{p}}$ est la vitesse de propagation dans la ligne et $l$ la distance du point $\mathrm{C}$ au point B ; dans la plupart des lasers $\tau_{\mathrm{p}}$ est de quelques ns ;

- $\tau_{\mathrm{m}}$ dépend principalement des caractéristiques 


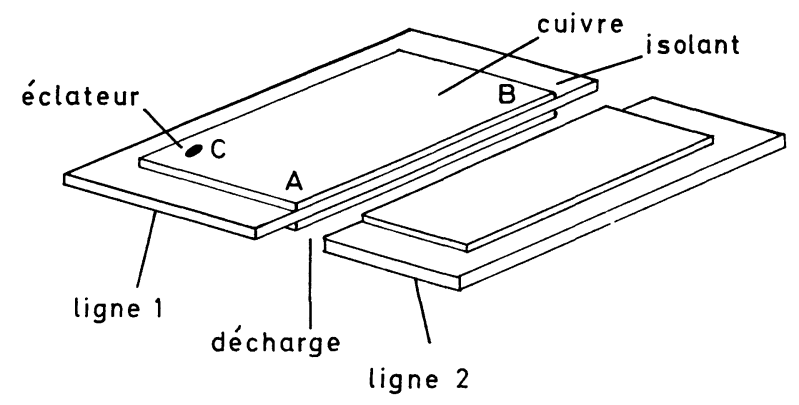

(a)

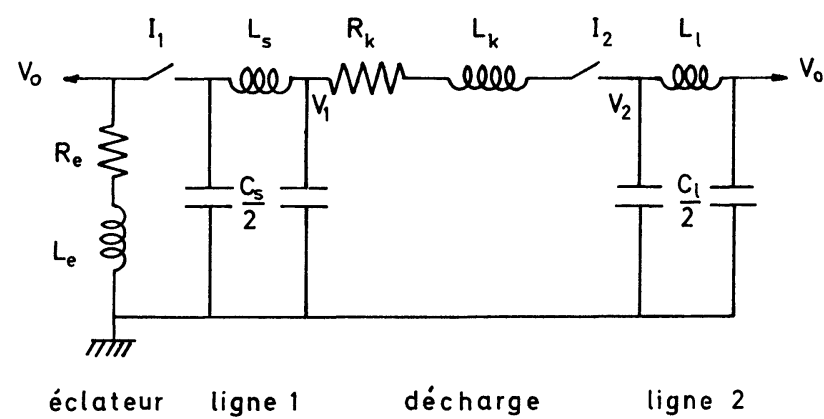

(b)

Fig. 1. - Schéma de principe et circuit équivalent de la structure d'un laser $\mathbf{N}_{2}$. Les deux lignes sont chargées à l'instant initial. L'interrupteur $\mathrm{I}_{1}$ est fermé à l'instant $t=0$, l'interrupteur $\mathrm{I}_{2}$ se ferme lorsque la tension aux bornes de la décharge est égale à la tension de claquage.

[Basic transversely excited $\mathbf{N}_{2}$ laser and equivalent circuit. The two lines are charged for $t=0$. Switch $\mathrm{I}_{1}$ is closed at $t=0$ and $I_{2}$ closes when the tension at the edges of the discharge is equal to the breakdown voltage.]

de l'éclateur et de l'impédance de la ligne. Actuellement il est difficile d'obtenir des éclateurs (avec des courants de quelques dizaines de kilo-ampères) avec des selfs inférieurs à $5 \mathrm{nH}$; de plus l'impédance de la ligne (qui doit être voisine de celle de la décharge) est de l'ordre de $0,1 \Omega$. En conséquence

$$
\tau_{\mathrm{m}} \simeq \frac{L}{R} \simeq 50 \mathrm{~ns}
$$

On voit que $\tau_{\mathrm{m}}$ est beaucoup plus grand que $\tau_{\mathrm{p}}$; par conséquent, il n'est pas juste de parler d'onde de tension dans un tel circuit, la tension étant identique en tout point du laminé au moment du claquage aux bornes de la décharge-laser.

Ce résultat est confirmé par l'étude du circuit équivalent [4] de la structure laser (Fig. 1b) ; ce circuit comprend :

- deux quadripoles en $\Pi$ qui représentent les deux lignes ;

- une résistance $R_{\mathrm{c}}$ et une self $L_{\mathrm{c}}$ en série qui représentent l'éclateur ;

- une résistance $R_{\mathrm{L}}$ et une self $L_{\mathrm{L}}$ en série pour représenter la décharge-laser; $R_{\mathrm{L}}$ a été choisie variable pour tenir compte du temps d'ionisation.
A l'instant initial les deux lignes sont chargées; l'éclateur se déclenche, la tension aux bornes de la ligne 1 décroît et il y a claquage dans la chambre-laser lorsque la tension aux bornes des électrodes $V_{\mathrm{k}}=V_{2}-V_{1}$ est égale à la tension de claquage du gaz à la pression donnée.

La figure 2 donne la tension et le courant dans la décharge laser. Malgré la faible valeur de la self de l'éclateur, le temps de montée $\tau_{\mathrm{m}}$ de $V_{\mathrm{k}}^{\prime}$, de plusieurs dizaines de ns, est environ 10 fois plus grand que le temps $\tau_{\mathrm{p}}$ de propagation (environ $3 \mathrm{~ns}$ ).

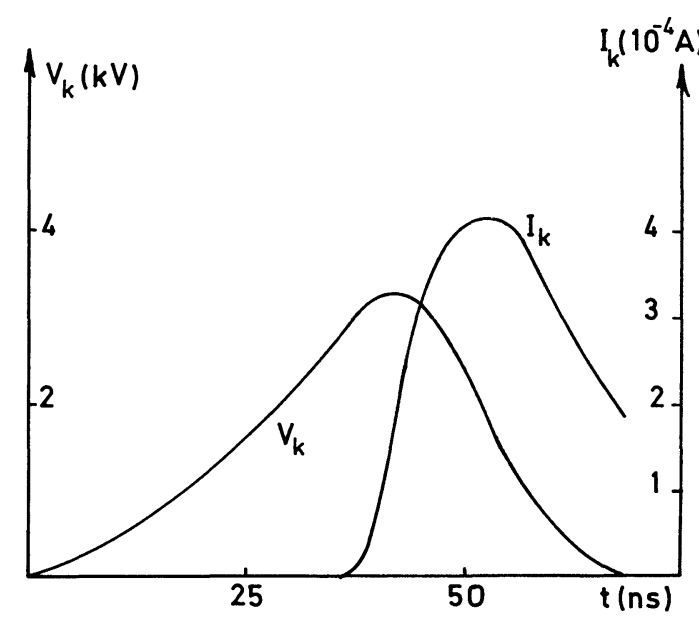

FIG. 2. - La tension aux bornes de la décharge et le courant en fonction du temps. $C_{\mathrm{s}}=C_{\mathrm{L}}=145 \mathrm{nF} ; L_{\mathrm{e}}=5 \mathrm{nH}$; $R_{\mathrm{e}}=0,1 \Omega ; L_{\mathrm{s}}=L_{\mathrm{L}}=0,1 \mathrm{nH} ; L_{\mathrm{k}}=1 \mathrm{nH}$. $R_{\mathrm{k}}$ décroît de $10 \Omega$ à $0,1 \Omega$ en 4 ns et reste constant pour $t>4$ ns. [Voltage waveform across the discharge and current. $C_{\mathrm{s}}=C_{\mathrm{L}}=145 \mathrm{nF} ; L_{\mathrm{e}}=5 \mathrm{nH} ; R_{\mathrm{e}}=0,1 \Omega ; L_{\mathrm{s}}=L_{\mathrm{L}}=0,1 \mathrm{nH} ;$ $L_{\mathrm{k}}=1 \mathrm{nH}$. $R_{\mathrm{k}}$ decreases from $10 \Omega$ to $1 \Omega$ for $t<4 \mathrm{~ns}$. $R_{\mathrm{k}}=0,1 \Omega$ for $t>4$ ns.]

\section{De ces résultats on peut conclure que :}

- puisque l'onde de tension qui naît au point $\mathrm{C}$ se réfléchit plusieurs fois sur les électrodes et sur les bords du laminé, il y a, au moment du claquage, une tension uniforme tout le long de l'électrode;

- quelle que soit la tension de charge des deux lignes, la tension aux bornes de la décharge laser sera déterminée uniquement par la pression du gaz et la distance interélectrode; $V_{\mathbf{k}}$ est toujours de l'ordre de $V_{\mathrm{c}}$, tension de claquage. En conséquence le rapport $E / p$ ( $E$ champ électrique, $p$ pression) est constant quelle que soit la tension de charge des lignes.

Pour que les structures comportant deux lignes deviennent intéressantes, il faudrait que l'impédance de la décharge laser (donc celles des deux lignes) soit plus grande de manière à diminuer le temps de montée $\tau_{\mathrm{m}}$. Néanmoins le temps de montée du courant dans la décharge laser n'est pas infiniment court. 
3. Une nouvelle structure laser. - 3.1 DesCRIPTION. - Compte tenu de ces résultats une nouvelle structure laser [5] a été mise au point (Fig. 3a). Elle permet de

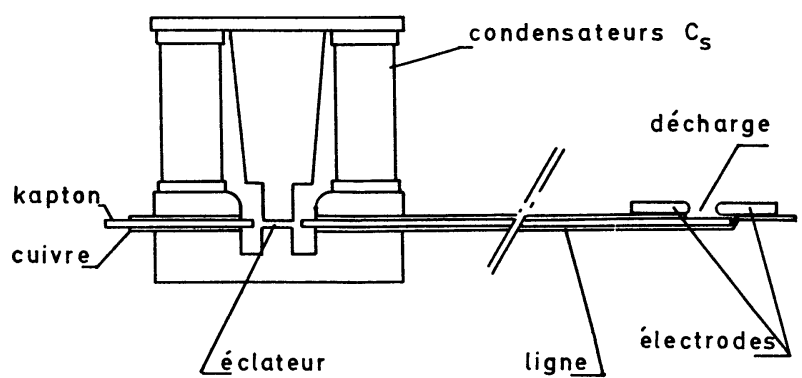

(a)

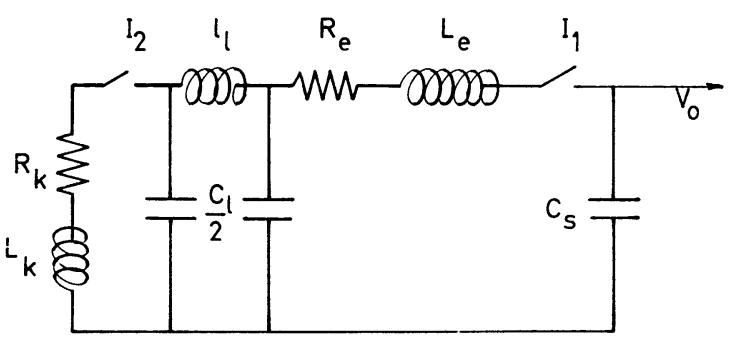

décharge ligne éclateur stockage

(b)

Fig. 3. - La nouvelle structure et son schéma équivalent. Uniquement $C_{\mathrm{s}}$ est chargé à l'instant initial. $\mathrm{I}_{2}$ se ferme lorsque $V_{1}=V_{\mathrm{c}}$.

[New structure and equivalent circuit. $C_{\mathrm{s}}$ is charged for $t=0$. $I_{2}$ closes when $V_{1}=V_{\mathrm{c}}$.]

découpler le stockage d'énergie de la mise en forme spatiale mais non temporelle comme dans le laser construit par M. Geller [6] de l'impulsion, et par suite de n'avoir jamais aux bornes du laminé une tension très supérieure à la tension de claquage. Elle comprend :

- des condensateurs discrets $C_{\mathrm{s}}$ qui sont chargés à l'instant initial à une tension $V_{0}$;

- un éclateur qui transfère l'énergie stockée dans $C_{\mathrm{s}}$ vers le laminé ;

- un condensateur plan (laminé) dont les deux plaques sont reliées directement aux électrodes de la décharge laser.

Le circuit équivalent (Fig. $3 b$ ) permet de calculer la tension aux bornes de la décharge et le courant (Fig. 4); on remarque la similitude des résultats et de ceux obtenus avec la précédente structure.

3.2 RéSUltats eXPÉRIMENTAUX. - Une des conséquences des calculs précédents est que la tension aux bornes de la décharge n'est que très légèrement supérieure à la tension $V_{\mathrm{c}}$ de claquage qui dépend du produit $(\mathrm{p} . d)$, où $d$ est la distance interélectrode.

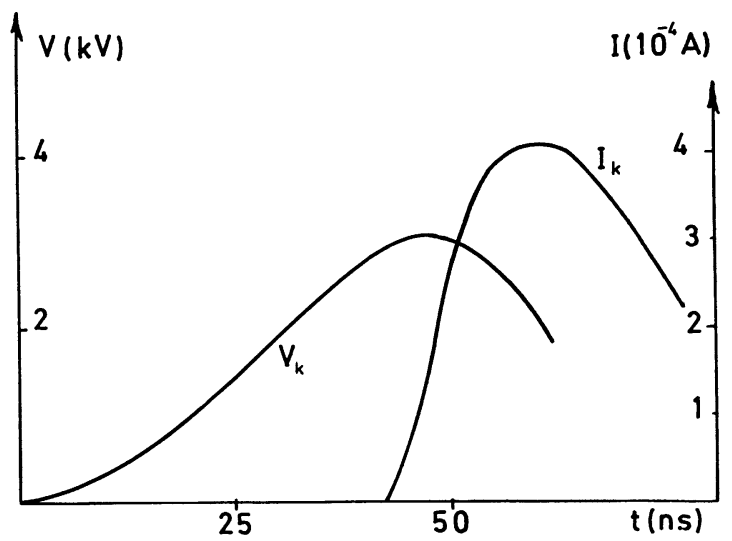

Fig. 4. - La tension aux bornes de la décharge et le courant en fonction du temps dans la nouvelle structure : $C_{\mathrm{s}}=C_{1}=145 \mathrm{nF}$; $L_{\mathrm{e}}=5 \mathrm{nH} ; R_{\mathrm{e}}=0,1 \Omega ; \quad L_{\mathrm{s}}=L_{\mathrm{L}} \simeq 0,1 \mathrm{nH} ; \quad L_{\mathrm{k}}=1 \mathrm{nH}$. $R_{\mathrm{k}}$ même valeur que pour la figure 2 .

[Voltage waveform across the discharge and current for the new structure : $C_{\mathrm{s}}=C_{\mathrm{L}}=145 \mathrm{nF} ; L_{\mathrm{e}}=5 \mathrm{nH} ; R_{\mathrm{e}}=0,1 \Omega$; $L_{\mathrm{s}}=L_{\mathrm{L}}=0,1 \mathrm{nH} ; L_{\mathrm{k}}=1 \mathrm{nH}$. $R_{\mathrm{k}}$ decreases from $10 \Omega$ to $1 \Omega$ for $t<4$ ns. $R_{\mathrm{k}}=0,1 \Omega$ for $t>4 \mathrm{~ns}$.

Par suite, à pression constante, la valeur du rapport $E / p$ où $E$ est le champ électrique, est presque constant quelle que soit la tension de charge $V_{0}$ aux bornes de $C_{\mathrm{s}}$; de plus dans le domaine de pression où ont été réalisées les expériences ce rapport est compris entre

$$
60<\frac{E}{p}(\text { volt } / \mathrm{cm} \cdot \mathrm{mmHg})<100 .
$$

Néanmoins on observe (Fig. 5) que

- pour une tension $V_{0}$, l'énergie laser passe par un maximum pour une valeur donnée de la pression ;

- pour une pression donnée, l'énergie laser croît en fonction de la tension $V_{0}$.

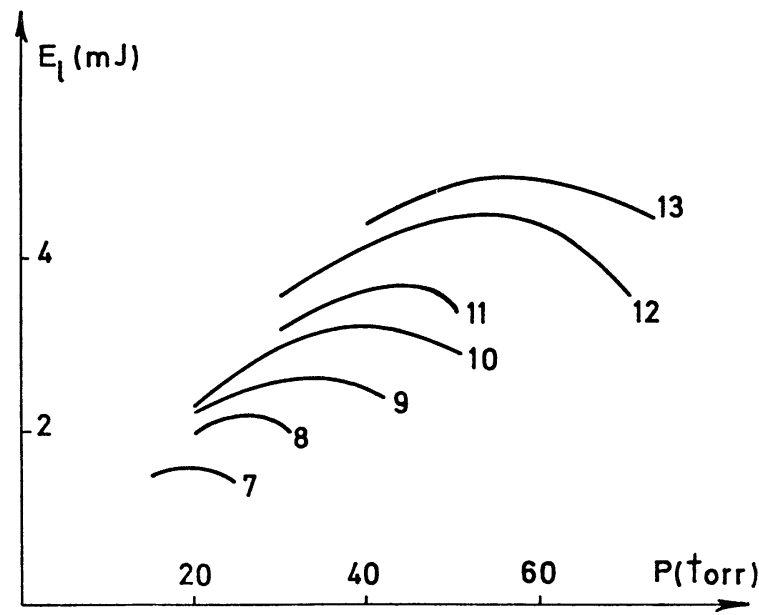

FIG. 5. - Energie laser en fonction de la pression pour diverses valeurs de la tension $V_{0}(\mathrm{en} \mathrm{kV}) . C_{\mathrm{s}}=60 \mathrm{nF} ; C_{\mathrm{L}}=145 \mathrm{nF}$; $d$ (distance interélectrode $)=1 \mathrm{~cm}$.

[Laser output power versus pressure for different voltages $V_{0}$. $C_{\mathrm{s}}=60 \mathrm{nF} ; C_{\mathrm{L}}=145 \mathrm{nF} ; d$ (electrode separation) $=1 \mathrm{~cm}$.] 
Une étude du circuit équivalent permet de montrer que le maximum d'énergie laser correspond au maximum de la pente $\mathrm{d} V_{\mathrm{k}} / \mathrm{d} t$ lorsque $V_{\mathrm{k}}=V_{\mathrm{c}}$ (qui dépend de la pression) c'est-à-dire approximativement lorsque la tension de claquage $V_{c}$ est de l'ordre de

$$
V_{\mathrm{c}}(p) \simeq V_{0} \frac{1}{1+\frac{C_{\mathrm{L}}}{C_{\mathrm{s}}}} .
$$

De plus si l'on trace (Fig. 6) pour une structure la courbe de la puissance laser en fonction de la pente $\mathrm{d} V_{\mathrm{k}} / \mathrm{d} t$ pour $V_{\mathrm{k}}=V_{\mathrm{c}}$, on remarque que l'on obtient une droite (qui ne passe pas par l'origine)

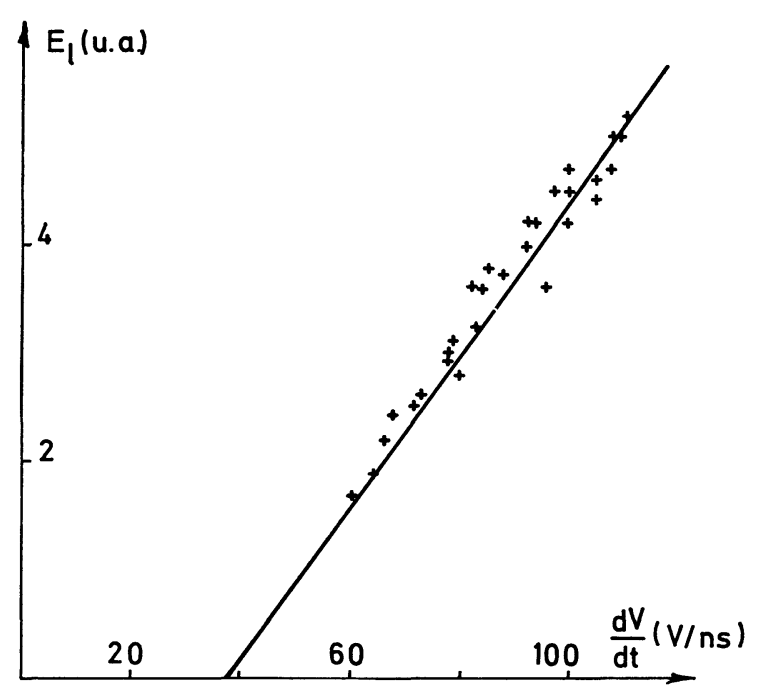

FIG. 6. - Energie laser en fonction de $\frac{\mathrm{d} V_{\mathrm{k}}}{\mathrm{d} t}\left(V_{\mathrm{k}}=V_{\mathrm{c}}\right)$. $C_{\mathrm{s}}=60 \mathrm{nF} ; C_{\mathrm{L}}=86 \mathrm{nF}$. La tension aux bornes de $C_{\mathrm{s}}$ varie entre 7 et $13 \mathrm{kV}$.

[Laser output power versus $\frac{\mathrm{d} V_{\mathrm{k}}}{\mathrm{d} t}\left(V_{\mathrm{k}}=V_{\mathrm{c}}\right) . C_{\mathrm{s}}=60 \mathrm{nF}$; $C_{\mathrm{L}}=86 \mathrm{nF}$. Voltage across $C_{\mathrm{s}}$ is varied between $7 \mathrm{kV}$ and $\left.13 \mathrm{kV}\right]$.

dont la pente dépend des composants de la structure (en particulier $C_{\mathrm{s}}$ et $C_{\mathrm{L}}$ ) ; cette figure regroupe les résultats obtenus en variant les deux paramètres tension et pression (Fig. 5). Cela veut dire que l'énergie laser dépend non pas de l'énergie qui se trouve dans la ligne $C_{\mathrm{L}}$ mais du courant qui circule dans $C_{\mathrm{L}}$ au moment du claquage (puisque $I=C_{\mathrm{L}} \frac{\mathrm{d} V}{\mathrm{~d} t}$ ) .

Deux conséquences importantes :

- pour une structure donnée (valeur de $C_{\mathrm{L}}$ et de $C_{\mathrm{s}}$ ) on peut connaître immédiatement (si la résistance de l'éclateur est faible) la courbe de l'énergie laser (en u. a) en fonction de la pression pour une tension de charge donnée. En particulier on peut connaître pour quelle pression l'énergie laser sera maximum en utilisant l'équation 2 ;

- pour cette valeur de $V_{\mathbf{k}}$, le rapport de l'énergie emmagasinée dans $C_{\mathrm{L}}$ par rapport à l'énergie initiale est au plus égale à

$$
r=\frac{C_{\mathrm{s}} C_{\mathrm{L}}}{\left(C_{\mathrm{s}}+C_{\mathrm{L}}\right)^{2}}
$$

Ce rapport est maximum pour $C_{\mathrm{s}}=C_{\mathrm{L}}$ et a pour valeur 0,25 ; il y a donc $75 \%$ de l'énergie qui est perdue dans le transfert.

On observe également que pour les maxima des courbes de la figure 5, l'énergie laser est proportionnelle à l'énergie électrique $\frac{1}{2} C_{\mathrm{s}} V_{0}^{2}$, ceci pour une structure donnée (Fig. 7). La comparaison entre

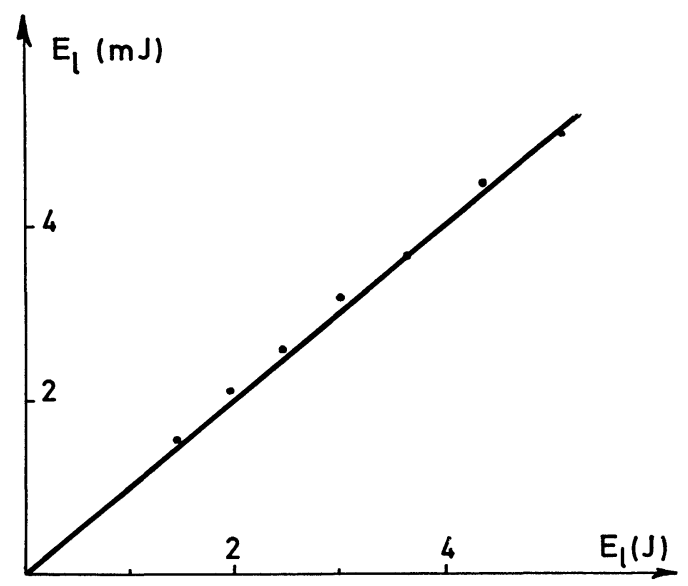

FIG. 7. - Les maxima de l'énergie laser (Fig. 5) en fonction de l'énergie électrique $\frac{1}{2} C_{\mathrm{s}} V_{0}^{2} . C_{\mathrm{s}}=60 \mathrm{nF} ; C_{\mathrm{L}}=145 \mathrm{nF}$. [Laser output power maxima versus electric energy $\frac{1}{2} C_{\mathrm{s}} V$ s. $C_{\mathrm{s}}=60 \mathrm{nF} ; C_{\mathrm{L}}=145 \mathrm{nF}$.]

diverses structures est complexe ; néanmoins il semble que l'énergie laser est proportionnelle à l'énergie $\frac{1}{2} C_{\mathrm{L}} V_{\mathrm{k}}^{2}$ au moment du claquage et lorsque $\mathrm{d} V_{\mathrm{k}} / \mathrm{d} t$ est maximum. Conséquence le meilleur rendement est obtenu lorsque $C_{\mathrm{L}}=C_{\mathrm{s}}$.

4. Conclusion. - Cette étude a montré l'importance du circuit électrique et des conditions de fonctionnement de la décharge sur les performances du laser $\mathrm{N}_{2}$ :

- perte de $75 \%$ de l'énergie dans le transfert entre $C_{\mathrm{s}}$ et $C_{\mathrm{L}}$;

- importance de la pente $\mathrm{d} V_{\mathrm{k}} / \mathrm{d} t$ au moment du claquage. Ce résultat peut sans doute s'expliquer de la manière suivante : la décharge n'est pas homogène mais formée de $n$ streamers $(2<n<8$ par $\mathrm{cm})$; au temps $t=t_{1}$, un streamer se forme et déclenche les autres (grâce à l'émission de photons du premier) ; pendant le temps de déclenchement des streamers on a avantage à avoir une augmentation rapide de la tension;

- la difficulté d'augmenter le rapport $E / p$ et donc de modifier le peuplement du niveau supérieur du laser. Il semble donc nécessaire pour agir sur le peuplement des niveaux d'utiliser des mélanges de gaz, ce qui permet de modifier la tension de claquage, donc le rapport $E / p$ et la mobilité des électrons. 


\section{Bibliographie}

[1] Willett, C. S., An introduction to gas lasers (Pergamon Press) p. 342. Une liste bibliographique assez complète est donnée dans ce livre.

[2] Godard, B., I. E. E. E. J. Quant. Electron. QE 10 (1974) 147.

[3] Par exemple : Von Bergmann, H. V., Hasson, V. et PreussLER, D., Appl. Phys. Lett. 27 (1975) 553.
[4] Schwah, A. et Hollinger, F., I. E. E. E. J. Quant. Electron. QE 12 (1976) 183.

[5] Huet, P., Milleon, H. et Leprince, P., Brevet ANVAR $n^{\circ} 7535084$ (1975).

[6] Geller, M., Altmann, D. E. et De Temple, T. A., Appl. Opt. 7 (1968) 2232. 